\title{
GABAergic System in Stress: Implications of GABAergic Neuron Subpopulations and the Gut-Vagus-Brain Pathway
}

\author{
Xueqin Hou, ${ }^{1}$ Cuiping Rong, ${ }^{2}$ Fugang Wang, ${ }^{1}$ Xiaoqian Liu, ${ }^{1}$ Yi Sun, ${ }^{1}$ \\ and Han-Ting Zhang $\mathbb{D D}^{3}$ \\ ${ }^{1}$ Institute of Pharmacology, Shandong First Medical University \& Shandong Academy of Medical Sciences, Tai'an, \\ Shandong 271016, China \\ ${ }^{2}$ The Second Clinical Medical College, Guangzhou University of Chinese Medicine, Guangzhou, Guangdong 510006, China \\ ${ }^{3}$ Departments of Neuroscience and Behavioral Medicine \& Psychiatry, The Rockefeller Neurosciences Institute, West Virginia \\ University Health Sciences Center, Morgantown, WV 26506, USA
}

Correspondence should be addressed to Han-Ting Zhang; hzhang@hsc.wvu.edu

Received 15 May 2020; Revised 3 July 2020; Accepted 6 July 2020; Published 1 August 2020

Academic Editor: Fushun Wang

Copyright ( 2020 Xueqin Hou et al. This is an open access article distributed under the Creative Commons Attribution License, which permits unrestricted use, distribution, and reproduction in any medium, provided the original work is properly cited.

Stress can cause a variety of central nervous system disorders, which are critically mediated by the $\gamma$-aminobutyric acid (GABA) system in various brain structures. GABAergic neurons have different subsets, some of which coexpress certain neuropeptides that can be found in the digestive system. Accumulating evidence demonstrates that the gut-brain axis, which is primarily regulated by the vagus nerve, is involved in stress, suggesting a communication between the "gut-vagusbrain" pathway and the GABAergic neuronal system. Here, we first summarize the evidence that the GABAergic system plays an essential role in stress responses. In addition, we review the effects of stress on different brain regions and GABAergic neuron subpopulations, including somatostatin, parvalbumin, ionotropic serotonin receptor 5-HT3a, cholecystokinin, neuropeptide $\mathrm{Y}$, and vasoactive intestinal peptide, with regard to signaling events, behavioral changes, and pathobiology of neuropsychiatric diseases. Finally, we discuss the gut-brain bidirectional communications and the connection of the GABAergic system and the gut-vagus-brain pathway.

\section{Introduction}

Stress is associated with various effects and mental disorders. Responses to stress vary from diet alteration to movement and sleep changes. Acute stress, such as trauma, can lead to rapid emotional changes and even result in long-term mental impairments. For instance, posttraumatic stress disorder (PTSD), a typical mental disorder, is often accompanied by depression and anxiety [1]. Chronic stress exposure, such as life stress (interpersonal loss, physical danger, humiliation, entrapment, role change/disruption, etc.), also increases depressive response and anxiety, and even triggers suicide in extreme cases [2-5]. Both acute and chronic stressinduced mental problems are associated with the $\gamma$-aminobutyric acid (GABA) system [6-9]. Gene polymorphism analy- sis of healthy subjects indicates that the GABA(A) $\alpha 6$ receptor subunit gene (GABRA6) polymorphism is responsive to psychological stress [10]. Therefore, agents targeting the GABAergic system are used to regulate depression, anxiety, or fear [11-13]. Interestingly, growing evidence has shown that gut-brain signals influence emotional behaviors [1416], and the gut-brain axis may be a possible target for treating stress-related disorders [17]. A recent review has summarized the psychophysiological effects of prebiotics and discussed the important roles of bacteria-gut-brain signals in psychobiotic activity [18]. In addition to the gut microbiome, neurotransmitters and neuropeptides are also involved in the gut-brain communications [19-21]. Some neurotransmitters and neuropeptides in the central nervous system (CNS) are involved in regulating the function of the 
digestive system [22-25]. Moreover, some neuropeptides are expressed in GABAergic neurons, which may be parts of the GABAergic system. In this review, we focus on how the GABAergic system impacts the gut-brain interaction in order to mediate stress-related disorders.

\section{GABAergic Neuron Signaling and Stress}

GABA is a major inhibitory neurotransmitter and is synthesized from the amino acid glutamate regulated by glutamate decarboxylases (GADs), including GAD1 and GAD2, whose genes encode GAD67 and GAD65 proteins, respectively [26]. GABAergic neurons are widely distributed in the CNS of mammals, and together with other GABA related factors, they compose the GABAergic system. The ventral medial prefrontal cortex (vmPFC) responds to the GABA reuptake inhibitor tiagabine [27] and is associated with fear responses and stress $[28,29]$, suggesting that the vmPFC GABAergic system plays a role in regulating stress-related emotion and responses. Additionally, many other GABAergic neuroncontaining brain structures (such as the hippocampus and amygdala) and GABA-associated signaling are also involved in the stress regulation $[30,31]$.

GABAergic neurons coexpress various proteins or neuropeptides, such as somatostatin, parvalbumin, ionotropic serotonin receptor 5-HT3a (5-HT3aR), cholecystokinin, neuropeptide Y (NPY), vasoactive intestinal peptide, calbindin, and calretinin [32-34]. In addition, $\sim 40 \%$ of GABAergic neurons are parvalbumin interneurons, $\sim 30 \%$ are somatostatin interneurons, and $\sim 30 \%$ are 5 -HT3aR interneurons in the neocortex [35], which make up the three major subtypes of GABAergic neurons. Other proteins or neuropeptides are expressed in different subtypes of GABAergic neurons. For example, cholecystokinin and vasoactive intestinal peptide may express in 5HT3aR interneurons, and NPY is colocalized with somatostatin interneurons [35].

The changes in subpopulations of GABAergic neurons vary in different brain areas under stress $[36,37]$. For example, long-term daily stress reduces the number of parvalbumin, calretinin, NPY, and somatostatin cells, but does not affect cholecystokinin and calbindin interneurons in the hippocampus [36]. Early life stress changes the structure and function of several brain regions, in addition to alterations of emotional behaviors and responses to stress in adults [38-40]. Exposure to long-term daily stress reduces calbindin neuron densities in the dorsolateral, medial, and ventral orbital cortex, but has no effect on cholecystokinin, NPY, parvalbumin, somatostatin, and calretinin neurons in any brain subregions in adult rats. Interestingly, enhanced density of cholecystokinin and NPY neurons in the ventral and lateral orbital cortices, respectively, is observed in stressresilient rats, suggesting that cholecystokinin and NPY in the orbitofrontal cortex may be involved in stress resilience [41]. Taken together, these results suggest a complex GABAergic network change under stress. Also, it raises a question of how to control the GABAergic network in order to regulate stress-induced emotional behaviors. Of those coexpressing markers in GABAergic neurons, cholecystoki- nin, NPY, and vasoactive intestinal peptide are also known as gut-related modulators and involved in the regulation of energy. Subsequently, how do they connect to GABA signaling to play a role in the regulation of stress?

2.1. Somatostatin. Somatostatin is a chemical marker of GABAergic neurons [33]. Somatostatin deficit is a common pathological characteristic in neurological disorders with emotional changes. In patients with schizophrenia and bipolar disorder, somatostatin-immunoreactive neurons are decreased in the lateral amygdala, which may affect responses to fear and anxiety [42]. Mice deficient in somatostatin exhibits high behavioral emotionality, increased basal plasma corticosterone, and decreased GABA-synthesizing enzyme GAD67 gene expression [43], indicating that somatostatin influences the GABA signal and stress response. Upon 2 weeks of chronic mild stress in rats, somatostatin-2 receptors are significantly upregulated in the medial habenula, while the plasma somatostatin levels are also increased, suggesting that somatostatin and its receptors are involved in the stress response [44]. Longer duration (e.g., 7 weeks) of chronic mild stress in rats can cause decreases in consumption of sucrose solution and changes in somatostatin-2 receptors in response to antidepressant treatment [45]. Moreover, selective inactivation of the $\gamma 2$ subunit gene of GABAA receptors in somatostatin-positive GABAergic interneurons (SSTCre: $\gamma 2(\mathrm{f} / \mathrm{f})$ mice) mimic the behavioral effects of antidepressant and anxiolytic drugs, suggesting that sustained increases in GABAergic transmission produce antidepressant-like behavior by disinhibiting somatostatin-positive GABAergic interneurons [46].

2.2. Parvalbumin. Parvalbumin is another chemical marker of GABAergic neurons. Parvalbumin and somatostatin interneurons play distinct roles in the medial entorhinal cortex [47], a critical brain region associated with contextual memory [48]. Besides, parvalbumin- and somatostatin-expressing interneurons in the mPFC also have different activity patterns (weak and strong targetdependent delay-period activity), as well as distinct stimulation effects in spatial working memory. For instance, parvalbumin interneurons are strongly inhibited by reward, while only a subtype of somatostatin interneurons is inhibited [49]. Thus, parvalbumin and somatostatin interneurons may function in different ways. Selectively silencing parvalbumin, but not somatostatin, interneurons in the infralimbic cortex eliminates ventral hippocampalmediated inhibition, while blocking infralimbic projectors reduces fear renewal [50], indicating that parvalbumin interneurons are involved in fear responses. Parvalbumin/GAD1 transgenic mice (silencing the GAD1) exhibit reduction of fear extinction, marked sensorimotor gating deficits, and elevated novelty-seeking [51]. Inhibition of parvalbumin interneurons disinhibits projection neurons from the prefrontal region and synchronizes their firing, resulting in fear [52]. After fear conditioning, parvalbumin interneurons show target- and region-selective plasticity in basolateral amygdala (BLA) subareas [53]. Together, parvalbumin interneurons regulate stress- 
induced fear, and fear affects the parvalbumin interneurons in return.

2.3. HT3aR. Among the serotonin (5-HT) receptors in mammals, the 5-HT3R is the only ligand-gated ion channel receptor for 5-HT. The 5-HT3aRs are found in cholecystokinin positive and vasoactive intestinal peptide positive GABAergic interneurons, and these 5-HT3aR-expressing vasoactive intestinal peptide/cholecystokinin interneurons receive serotonergic and cholinergic fast synaptic transmission [54]. Furthermore, coexpression of 5-HT3aR and central calbindin 1 cannabinoid receptors have been detected in GABAergic neurons in the anterior olfactory nucleus, the cerebral cortex, hippocampus, dentate gyrus, subiculum, entorhinal cortex, and amygdala $[55,56]$. Interestingly, the activation of 5HT3 receptors by serotonin causes GABA release, whereas stimulation of calbindin 1 receptors by cannabinoids inhibits GABA release, indicating opposing effects on GABA neurotransmission [55]. The amygdala has been known to be involved in the regulation of emotion. A moderate density of 5-HT3aR neurons are found in the amygdalar basolateral nuclear complex, and almost all 5-HT3aR neurons are GABA positive. Therefore, serotonin may activate 5 - $\mathrm{HT} 3$ receptors in the 5-HT3aR positive GABAergic neurons in the amygdala and lead to GABA release, resulting in emotional changes under stress.

2.4. Cholecystokinin. Cholecystokinin is a peptide hormone produced by enteroendocrine cells of the small intestine and released into the blood. Cholecystokinin is also widely distributed throughout the CNS, with high levels in the limbic system. The sulfated octapeptide, cholecystokinin- $8 \mathrm{~S}$, is the major biologically active form of cholecystokinin in the CNS [57]. Intraperitoneal injections of cholecystokinin-8 enhance c-Fos (an immediate-early gene) expression in the dorsal CA3 and dentate gyrus of the hippocampus [58], indicating that cholecystokinin-8 activates neurons in the hippocampus. In the dentate gyrus, the activation of presynaptic 5HT1B receptors in cholecystokinin interneurons inhibits GABA release and further disinhibits parvalbumin interneurons, leading to reduction of the granule cells activity. Furthermore, the inhibition of cholecystokinin neurons exhibits antidepressant-like effects on behavior, similar to selective serotonin reuptake inhibitors [59]. Thus, the activation of cholecystokinin neurons affects GABA release and depressant-like behavior.

Cholecystokinin-4, another form of cholecystokinin, has been known to induce panic attacks. In a double-blind, placebo-controlled study, 26 of 30 subjects exhibited obvious panic responses when they were challenged with cholecystokinin-4 [60]. Subjects who were treated with anxiolytics alprazolam prior to the rechallenge of cholecystokinin- 4 showed a significant reduction of the panic-related scale scores and reported symptoms, as well as lower adrenocorticotropic-hormone and cortisol release. Because slow GABABR-mediated inhibitory postsynaptic currents were recorded in most cholecystokinin interneurons [61], it is possible that cholecystokinin interacts with the GABAergic system. Systemic activation of cholecystokinin-
GABA neurons by clozapine-N-oxide in triple transgenic cholecystokinin-GABA/hM3Dq mice, in which about $22 \%$ of GABAergic neurons in the hippocampus and $19 \%$ in the prefrontal cortex are cholecystokinin-GABA neurons, not only enhanced contextual fear conditioning/discrimination, social/object recognition, and puzzle box performance, but also enhanced anxiety in the elevated plus maze [62].

2.5. NPY. NPY is a peptide derived from the brain and sympathetic nerves and involved in various functions in both the peripheral and central nervous systems. In the periphery, NPY is mainly released from the sympathetic nerves and serves as a regulator of fat growth [63]. In the brain, it is produced in various regions (such as the hypothalamus and amygdala) and is implicated in multiple functions, including energy homeostasis, food intake, metabolism, and stress response [64-67]. Stress can increase NPY expression in the brain [67]. Also, stress response and emotion can be affected by human NPY expression, as lower haplotype-driven NPY expression is related to higher emotion-induced activation of the amygdala [68]. Thus, NPY is thought to have stress-relieving and anxiolytic properties [69]. Chronic unpredictable stress for 5 weeks has been shown to reduce GAD67 protein levels in the prefrontal cortex and hippocampus in rats, without changing GAD65 protein expression. Additionally, the protein and RNA levels of somatostatin and NPY are also decreased following stress exposure, suggesting these subsets of GABAergic neurons may be sensitive to chronic stress [70]. NPY is colocalized with somatostatin interneurons in the brain [35]. In the BLA, somatostatin interneurons express NPY2-receptors, some of which coexpress NPY; stimulating BLA NPY2-receptors reduces tonic GABA release onto local principal neurons [30]. A combination of stress and high-fat diet activates central amygdala NPY neurons, resulting in increased feeding and reduced energy expenditure [67].

2.6. Vasoactive Intestinal Peptide. Vasoactive intestinal peptide, a gut hormone regulating energy metabolism [71, 72], is produced in many tissues, such as the gut and the hypothalamic suprachiasmatic nucleus in the brain $[71,73]$. In the CNS, neocortical vasoactive intestinal peptide positive neurons are one subpopulation of GABAergic interneurons [74]. Vasoactive intestinal peptide increases GABA release in the hippocampus without changing glutamate release. Concerted synaptic action of vasoactive intestinal peptide causes disinhibition of pyramidal cell dendrites and enhances GABAergic transmission [75]. The connections between different types of GABAergic neurons result in disinhibitory effects. For example, in the primary somatosensory cortex, most of the parvalbumin cells are innervated by vasoactive intestinal peptide neurons [76]. Therefore, neocortical vasoactive intestinal peptide positive GABAergic neurons send outputs onto other interneurons or principal neurons and display a disinhibitory effect [74]. Vasoactive intestinal peptide modulates hippocampal synaptic GABAergic transmission via activation of two vasoactive intestinal peptide receptors, i.e., VPAC1 and VPAC2 receptors, which, 
however, possess opposite effects on GABA release, as activation of VPAC1 or VPAC2 receptors inhibits or enhances GABA release, respectively [77]. Together, vasoactive intestinal peptide can affect GABAergic neurons, GABA level, and GAD expression [78, 79], which may further influence the stress-related behaviors.

\section{Crosstalk between the GABAergic System and the Gut-Brain Pathway in Stress}

3.1. The Vagus Nerve-Mediated Gut-Brain Pathway. The vagus nerve is an important neuronal component of the bidirectional communication of the gut-brain axis [80]. In addition to regulating the ingestive behavior, vagal afferent signaling has been implicated in the modulation of mood and affect, such as motivation and depression [81, 82]. Abdominal vagal afferents in rats display anhedonic behavior and increase behavioral despair [82]. It has been reported that disrupted vagal afferent signaling by subdiaphragmatic vagal deafferentation results in brain transcriptional changes in functional networks associated with schizophrenia, as well as dopamine alteration in the nucleus accumbens [83]. In another study, subdiaphragmatic vagal deafferentation rats exhibited a reduction in innate anxiety-like behavior assessed by open field test, elevated plus maze test, and food neophobia test, whereas their learning auditory-cued fear was increased [84]. Furthermore, these behavioral changes were related to the alterations of GABA and noradrenaline levels in the limbic system, without functional changes in the hypothalamus-pituitary-adrenal grand stress [84]. It suggests that vagal afferents may connect with the limbic system and affect the GABAergic system in the CNS. Selective ablation of gastrointestinal vagal sensory/afferent by saporin-based lesion impaired hippocampus-dependent behaviors in rats, indicating that vagus-mediated gut signaling, activates the hippocampus. Further monosynaptic and multisynaptic virus-based tracing investigation revealed a "medial nucleus tractus solitarius-medial septum-dorsal hippocampus glutamatergic neurons" connection, suggesting the existence of "gut-vagus-brainstem-septum-hippocampus" pathway [58]. Two types of vagal sensory neurons have been found to target the nucleus of the solitary tract (NTS) [85]. Moreover, various brain regions have been identified to be connected with the gut via the vagus nerve. Following the injection of pathological $\alpha$-syn preformed fibrils into the duodenal and pyloric muscularis layer, pathologic $\alpha$-syn could spread to the dorsal motor nucleus (DMN), caudal portions of the hindbrain (including the locus coeruleus), BLA, dorsal raphe nucleus (DRN), and the substantia nigra pars compacta (SNC). In addition, this gut-to-brain spread could be prevented by truncal vagotomy and $\alpha$-syn deficiency [86]. This study supports the idea that the vagus nerve directly mediates the communication from the gut to the brain.

In the NTS, cholecystokinin-containing neurons, activation of which reduces appetite, are responsive to nutritional state and send projections to the paraventricular nucleus of the hypothalamus (PVH) [87]. The PVH also projects directly to the NTS [88], thereby establishing a connection from the brain to gut linked by the vagus nerve $[80,89]$.
Thus, the central autonomic network integrates the vagus nerve mediated visceral information and regulates the hypothalamic-pituitary-adrenal (HPA) axis [90], which is implicated in stress-related disorders [91, 92]. Moreover, cholecystokinin- 4 administration could alter anxiety-like behavior and the HPA axis hormones such as corticosterone in rats exposed to early life stress [93].

To sum up, the vagus nerve-mediated gut-brain pathways at least involve "gut-vagus-NTS-septum-hippocampus" and "gut-vagus-DMN-hindbrain/BLA/DRN/SNC" pathways. The "NTS-PVH" loop might be a potential connection that regulates the "up-down" and "down-up" transmission. These complex neural pathways involve various stressrelated brain regions, within which GABA signals play a crucial role. Alongside the gut-vagus-brain pathway, gutassociated factors, including cholecystokinin, NPY, and vasoactive intestinal peptide, act as modulators of GABA signaling, so as to regulate stress. Thus, we further discuss the crosstalk between the GABAergic system and the vagus mediated gut-brain pathway, especially the link with the hippocampus, amygdala, and hypothalamus, as well as related neural network.

3.2. The Crosstalk between the GABAergic System and the Vagus Nerve-Mediated Gut-Brain Pathways. The hippocampus is an important brain structure involved in various neural circuits and functions. Exposure to chronic stress has been shown to be accompanied by rising GABA levels in the dorsal hippocampus [94]. However, different stressors may cause distinct changes in hippocampal extracellular GABA levels; for instance, a novel environment increases GABA whereas forced swimming reduces GABA [7]. Interestingly, chronic stress affects specific GABAergic neuronal subpopulations in the hippocampus, including parvalbumin, calretinin, NPY, and somatostatin neurons, but not cholecystokinin and calbindin interneurons $[8,36]$. The hippocampus receives inputs from the septum [95] and generates theta oscillations linked to multiple processes, including affect and locomotion $[96,97]$. The septum receives inputs from the median raphe nucleus, in which inhibition of the GABAergic pathway affects theta oscillations and decreases anxiety [98]. Both the lateral and medial septum GABAA receptor signal can influence the hippocampal theta frequency, and the GABAA receptor agonist muscimol infused in the dorsal lateral septum reduces anxiety-like behavior [99]. Infusion of the GABAB receptor agonist baclofen into the lateral septum reduces stress-induced anorectic effect while increases sucrose intake [100]. It has been shown that early-life stress reduces GAD67 in the lateral septum [101]. These results suggest that the lateral septum GABAergic system is related to stress and food intake. Moreover, somatostatin interneurons in the dorsal lateral septum receive inputs from hippocampal CA3 directly [102], thereby forming a feedback loop between the hippocampus and the septum. The medial septum sends both GABAergic and glutamatergic outputs to the lateral habenula, which affects the aversion [103]. In addition, somatostatin interneurons in the hippocampus can be selectively inhibited by GABAergic neurons from the nucleus incertus, modulating of which 
can shift the hippocampal network state and modify fear [31]. Overall, the median raphe nucleus projects to the septum, which projects to the hippocampus and lateral habenula, and the nucleus incertus projects to the hippocampus, thus forming a complex neural network associated with stress.

Amygdala is associated with stress and fear regulation [104-107]. In patients with schizophrenia and bipolar disorder, somatostatin positive neurons decreased in the amygdala [42]. Selective activation of NPY neurons in the central amygdala (CeA) leads to increased food intake and decreased energy expenditure under stress [67]. GABAergic serotonin receptor 2a-expressing neurons in the CeA modulate food consumption [108]. Furthermore, BLA to CeA neural circuit also mediates appetitive behaviors [109]. Thus, the BLA-CeA microcircuit within the amygdala plays a potential role in regulating stress and stress-induced appetitive behaviors. In the BLA, 5-HT3aR positive GABAergic neurons are found, and the main coexpressing marker is cholecystokinin, very few express calretinin, vasoactive intestinal peptide, or parvalbumin, and none expresses somatostatin or calbindin [110]. Another study also shows that vasoactive intestinal peptide interneurons are found in the mouse BLA [111]. Dopamine in the BLA selectively suppressed GABAergic transmission from parvalbumin interneurons to principal neurons but not to interneurons [112]. Activation of BLA NPY2-receptors reduces tonic GABA release onto BLA principal neurons and increases anxiety [30]. Selective activation of the BLA-mPFC input provides a safety-signaling mechanism whereby the mPFC taps into the microcircuitry of the amygdala to reduce fear [113]. In general, BLA neurons project to the CeA and mPFC, and the GABA pathways within these circuits are implicated in stress regulation through multiple mechanisms.

The $\mathrm{mPFC}$ is an important brain region involved in the emotional memories. By using a rat model for depression, researchers examined the effect of stress on GABAergic system changes in the mPFC [114]. Nine weeks of chronic mild stress exposure has been shown to decrease the amount of cholecystokinin, calretinin, and parvalbumin-positive GABAergic neurons in the mPFC. In contrast, NPYpositive neurons are increased in the entire MPFC in stressresilient rats. Moreover, the object-place paired-associate learning is impaired in stress-susceptible rats, suggesting that fronto-limbic GABAergic dysfunctions may contribute to emotional changes in depression [114]. In addition, chronic stress increases presynaptic GABA release, which is accompanied by increased inhibition onto prefrontal glutamatergic output neurons, leading to a reduced effect on modulating stress-related behavior [115]. The frontal cortex subregion cingulate projects to the primary visual cortex and affects visual discrimination. These long-range projections induce synaptic disinhibition of pyramidal neurons through local GABAergic neurons microcircuit, including vasoactive intestinal peptide, somatostatin, and parvalbumin-positive GABAergic interneurons [116].

The hypothalamus is a component of the HPA axis. Neurons in the hypothalamus subarea PVH produce corticotropin-releasing hormone $(\mathrm{CRH})$ involved in endo- crine stress response. GABAergic neurons projecting to the PVH regulate the excitability of CRH neurons [117]. Following adrenalectomy in rats, the synthetic and secretory activities of CRH neurons are increased, and a higher number of GABA-CRH synaptic contacts are detected in the PVH [118], suggesting a connection between the GABAergic system and the HPA axis. Moreover, a population of CRH positive GABAergic long-range-projecting neurons in the extended amygdala innervates the ventral tegmental area, and the chronic lack of CRH from this type of neurons produces anxiety [119]. Therefore, the GABAergic system may regulate anxiety-like behavior through the HPA axis and related networks. As described previously, there is a "NTS-PVH" loop linking to the "gut-vagus-NTS-septumhippocampus" pathway. Intraperitoneal injection of cholecystokinin-8S increases the amount of activated neurons in the NTS and PVH [120], while activating the NTS cholecystokinin axon terminals within the PVH affects appetite [87, 121], suggesting the effects on the gut pathway. The vagus nerve stimulation reduces the $\mathrm{CRH} /$ adrenocorticotropic hormone responses in the depressed subjects [122], suggesting the potential connection between the vagus and the HPA axis. Collectively, the gut and vagus pathways are related to the HPA axis, at least in part, through the "NTS-PVH".

3.3. Gut Microbiome in the GABAergic System and Vagal Communication. Growing evidence has shown that gut microbiome is involved in regulating stress-related behaviors and brain functions. Stress-associated anxiety- and depression-related behaviors are prevented by treatment with Lactobacillus paracasei Lpc-37, Lactobacillus plantarum LP12407, Lactobacillus plantarum LP12418, and Lactobacillus plantarum LP12151 [123]. Lactobacillus plantarum LP12418 can normalize the stress-induced reduction in adrenocorticotropic hormone [123]. Following probiotic bacterium Lactobacillus rhamnosus (JB-1) treatment, GABA levels are increased in the brain [124], and expression of $\operatorname{GABA}(\mathrm{B} 1 \beta)$ and $\operatorname{GABA}(\mathrm{A} \alpha 2)$ is changed in several brain regions, including the hippocampus (lower $\operatorname{GABA}(\mathrm{B} 1 \beta)$, higher $\operatorname{GABA}(\mathrm{A} \alpha 2)$ ), amygdala (lower $\operatorname{GABA}(\mathrm{B} 1 \beta)$ and $\operatorname{GABA}(\mathrm{A} \alpha 2))$, and prefrontal cortex (lower $\operatorname{GABA}(\mathrm{A} \alpha 2)$ ) [14]. One possible reason might be because many strains of Lactobacillus and Bifidobacterium are able to produce large quantities of GABA and activate GABA producing pathways $[125,126]$. Moreover, Lactobacillus rhamnosus (JB-1) decreases stress-induced corticosterone and anxiety- and depression-related behavior, while no effects are found in vagotomized mice [14]. Similarly, Lactobacillus plantarum LP12418 also changes the expression of $\operatorname{GABA}(\mathrm{A} \alpha 2)$ and $\operatorname{GABA}(\mathrm{B} 1 \beta)$ in the prefrontal cortex [123]. Lactobacillus casei strain Shirota not only suppresses stress-induced increases in glucocorticoids both in subjects and in rats but also stimulates vagal afferent activity and suppresses stress-induced activation of CRF cells in the PVH [127]. However, the effects of Lactobacillus rhamnosus (JB-1) were still unsatisfying in modifying stress-related measures and HPA response in male subjects in a clinical trial [128]. Overall, gut microbiome may play 


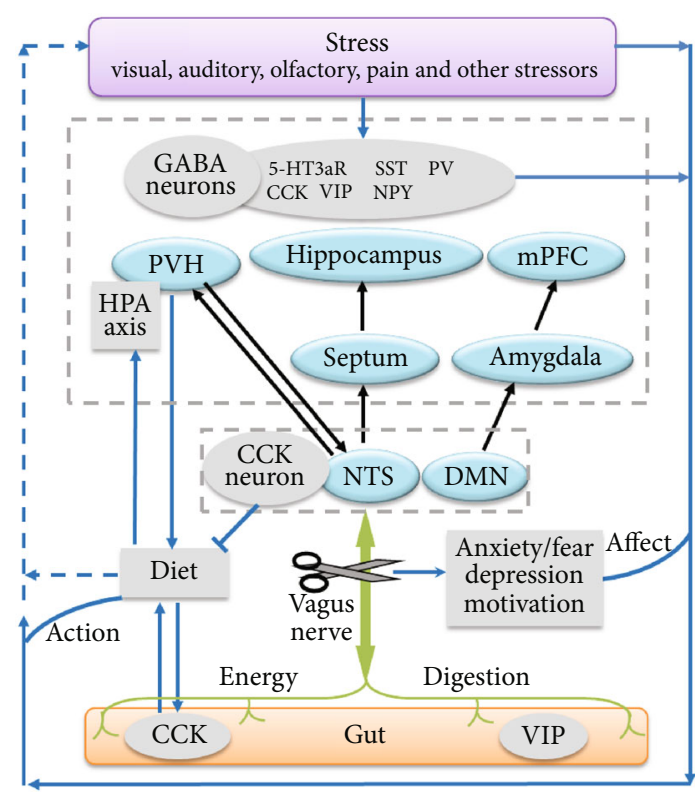

FIgURE 1: Potential role of crosstalk between the GABAergic system and the gut-vagus-brain pathway in stress. GABA, $\gamma$-aminobutyric acid; 5-HT3aR, serotonin receptor 5-HT3a; CCK, cholecystokinin; VIP, vasoactive intestinal peptide; SST, somatostatin; NPY, neuropeptide Y; PV, parvalbumin; $\mathrm{mPFC}$, medial prefrontal cortex; PVH, paraventricular nucleus of the hypothalamus; NTS, nucleus of the solitary tract; DMN, dorsal motor nucleus; HPA, hypothalamic-pituitary-adrenal.

an important role in regulating stress-related behavior through the GABAergic system and the gut-vagus-brain pathway.

\section{Conclusion and Future Perspectives}

Stress can cause various mental changes and reactions, which may be attributed to multiple changes in the body, including the brain structure and related circuitry pathway. Several gut-related modulators, such as cholecystokinin, NPY, and vasoactive intestinal peptide, not only express in the digestive system but also exist in the CNS and colocalize with GABAergic neurons. In addition to regulating the diet, they are also implicated in stress, which involves various brain structures. Stress may alter gut-associated behavior, such as increased or decreased food intake $[129,130]$, which can further affect the gut hormone release [131]. The vagus nerve connects the gut with the brain bidirectionally, thereby establishing a gutvagus-brain pathway. Thus, the crosstalk between the GABAergic system and the gut-vagus-brain pathway may play a potential role in stress (Figure 1).

Given that specific neuron types can be manipulated with chemogenetic and optogenetic approaches, the roles of different GABAergic neuron subgroups or relative cellular and molecular signals in stress can be further investigated. This may help find out more potential therapeutic targets for the treatment of stress-related CNS disorders, such as PTSD. It has been known that individuals with low plasma GABA levels are more susceptible to PTSD
[132], which is commonly accompanied with functional gastrointestinal disorders [133]. Recent evidence indicates that gut microbiome is associated with stress-induced behaviors [134, 135]. Vagus nerve stimulation may improve PTSD-like symptoms [136]. Therefore, manipulation of the gut-vagus-brain pathway may have therapeutic potential for treating PTSD. However, the complex neuronal markers may lead to various functions of each GABAergic neuron subset in different brain regions or even in different subareas of the same region. In addition, the complicated connections between the GABAergic system and the gut-vagus-brain pathway may play a potential role in regulating stress. Further studies are needed to increase the target and region selectivity, which appears to be a challenge to the development of novel drugs or approaches for the treatment of stress-induced CNS disorders.

\section{Conflicts of Interest}

The authors declare no conflict of interest.

\section{Acknowledgments}

This work was supported by research grants from the Key Research and Development Project of Shandong Province (2019GSF108069), the National Natural Science Foundation of China (81703901), the Development Project of Traditional Chinese Medicine Science and Technology in Shandong Province (No. 2019-0361), the Academic Promotion Project of Shandong First Medical University (2019LJ003), and the Youth Innovation Team of Shandong Universities (2019KJK001).

\section{References}

[1] M. Başoğlu, M. Paker, E. Özmen, Ö. Taşdemir, and D. Şahin, "Factors related to long-term traumatic stress responses in survivors of torture in Turkey," JAMA: The Journal of the American Medical Association, vol. 272, no. 5, pp. 357-363, 1994.

[2] S. E. Mayer, N. L. Lopez-Duran, S. Sen, and J. L. Abelson, "Chronic stress, hair cortisol and depression: A prospective and longitudinal study of medical internship," Psychoneuroendocrinology, vol. 92, pp. 57-65, 2018.

[3] J. G. Stewart, G. S. Shields, E. C. Esposito et al., "Life Stress and Suicide in Adolescents," Journal of Abnormal Child Psychology, vol. 47, no. 10, pp. 1707-1722, 2019.

[4] J. L. Lukkes, S. Meda, B. S. Thompson, N. Freund, and S. L. Andersen, "Early life stress and later peer distress on depressive behavior in adolescent female rats: Effects of a novel intervention on GABA and D2 receptors," Behavioural Brain Research, vol. 330, pp. 37-45, 2017.

[5] D. M. Fergusson, L. J. Horwood, A. L. Miller, and M. A. Kennedy, "Life stress, 5-HTTLPR and mental disorder: findings from a 30-year longitudinal study," The British Journal of Psychiatry, vol. 198, no. 2, pp. 129-135, 2011.

[6] X. Gonda, P. Petschner, N. Eszlari et al., "Effects of Different Stressors Are Modulated by Different Neurobiological Systems: The Role of GABA-A Versus CB1 Receptor Gene 
Variants in Anxiety and Depression," Frontiers in Cellular Neuroscience, vol. 13, 2019.

[7] L. de Groote and A. C. E. Linthorst, "Exposure to novelty and forced swimming evoke stressor-dependent changes in extracellular GABA in the rat hippocampus," Neuroscience, vol. 148, no. 3, pp. 794-805, 2007.

[8] W. Hu, M. Zhang, B. Czéh, G. Flügge, and W. Zhang, "Stress Impairs GABAergic Network Function in the Hippocampus by Activating Nongenomic Glucocorticoid Receptors and Affecting the Integrity of the Parvalbumin-Expressing Neuronal Network," Neuropsychopharmacology, vol. 35, no. 8, pp. 1693-1707, 2010.

[9] L. C. Houtepen, R. R. Schür, J. P. Wijnen et al., "Acute stress effects on GABA and glutamate levels in the prefrontal cortex: A 7T $1 \mathrm{H}$ magnetic resonance spectroscopy study," NeuroImage: Clinical, vol. 14, pp. 195-200, 2017.

[10] M. Uhart, M. E. McCaul, L. M. Oswald, L. Choi, and G. S. Wand, "GABRA6 gene polymorphism and an attenuated stress response," Molecular Psychiatry, vol. 9, no. 11, pp. 998-1006, 2004.

[11] M. Bixo, K. Ekberg, I. S. Poromaa et al., "Treatment of premenstrual dysphoric disorder with the GABA A receptor modulating steroid antagonist Sepranolone (UC1010)-A randomized controlled trial," Psychoneuroendocrinology, vol. 80, pp. 46-55, 2017.

[12] G. M. Gafford, J. D. Guo, E. I. Flandreau, R. Hazra, D. G. Rainnie, and K. J. Ressler, "Cell-type specific deletion of GABA(A) 1 in corticotropin-releasing factor-containing neurons enhances anxiety and disrupts fear extinction," Proceedings of the National Academy of Sciences, vol. 109, no. 40, pp. 16330-16335, 2012.

[13] P. Zwanzger, D. Eser, C. Nothdurfter et al., "Effects of the GABA-reuptake inhibitor tiagabine on panic and anxiety in patients with panic disorder," Pharmacopsychiatry, vol. 42, no. 6, pp. 266-269, 2009.

[14] J. A. Bravo, P. Forsythe, M. V. Chew et al., "Ingestion of Lactobacillus strain regulates emotional behavior and central GABA receptor expression in a mouse via the vagus nerve," Proceedings of the National Academy of Sciences, vol. 108, no. 38, pp. 16050-16055, 2011.

[15] M. Soto, C. Herzog, J. A. Pacheco et al., "Gut microbiota modulate neurobehavior through changes in brain insulin sensitivity and metabolism," Molecular Psychiatry, vol. 23, no. 12, pp. 2287-2301, 2018.

[16] P. Zheng, B. Zeng, M. Liu et al., "The gut microbiome from patients with schizophrenia modulates the glutamateglutamine-GABA cycle and schizophrenia-relevant behaviors in mice," Science Advances, vol. 5, no. 2, article eaau8317, 2019.

[17] L. V. Scott, G. Clarke, and T. G. Dinan, "The Brain-Gut Axis: A Target for Treating Stress-Related Disorders," Inflammation in Psychiatry, vol. 28, pp. 90-99, 2013.

[18] A. Sarkar, S. M. Lehto, S. Harty, T. G. Dinan, J. F. Cryan, and P. W. J. Burnet, "Psychobiotics and the Manipulation of Bacteria-Gut-Brain Signals," Trends in Neurosciences, vol. 39, no. 11, pp. 763-781, 2016.

[19] P. Holzer and A. Farzi, "Neuropeptides and the MicrobiotaGut-Brain Axis," Microbial Endocrinology: The MicrobiotaGut-Brain Axis in Health and Disease, pp. 195-219, 2014.

[20] R. Mittal, L. H. Debs, A. P. Patel et al., "Neurotransmitters: The Critical Modulators Regulating Gut-Brain Axis," Journal of Cellular Physiology, vol. 232, no. 9, pp. 2359-2372, 2017.
[21] K. G. Jameson and E. Y. Hsiao, "Linking the Gut Microbiota to a Brain Neurotransmitter," Trends in Neurosciences, vol. 41, no. 7, pp. 413-414, 2018.

[22] D. D. Lam and L. K. Heisler, "Serotonin and energy balance: molecular mechanisms and implications for type 2 diabetes," Expert Reviews in Molecular Medicine, vol. 9, no. 5, pp. 1-24, 2007

[23] Q. Tong, C.-P. Ye, J. E. Jones, J. K. Elmquist, and B. B. Lowell, "Synaptic release of GABA by AgRP neurons is required for normal regulation of energy balance," Nature Neuroscience, vol. 11, no. 9, pp. 998-1000, 2008.

[24] H. Wang, G. Astarita, M. D. Taussig et al., "Deficiency of Lipoprotein Lipase in Neurons Modifies the Regulation of Energy Balance and Leads to Obesity," Cell Metabolism, vol. 13, no. 1, pp. 105-113, 2011

[25] S. X. Luo, J. Huang, Q. Li et al., "Regulation of feeding by somatostatin neurons in the tuberal nucleus," Science, vol. 361, no. 6397, pp. 76-81, 2018.

[26] B. P. Grone and K. P. Maruska, "Three Distinct Glutamate Decarboxylase Genes in Vertebrates," Scientific Reports, vol. 6, no. 1, 2016.

[27] K. C. Evans, N. M. Simon, D. D. Dougherty et al., "A PET Study of Tiagabine Treatment Implicates Ventral Medial Prefrontal Cortex in Generalized Social Anxiety Disorder," Neuropsychopharmacology, vol. 34, no. 2, pp. 390-398, 2009.

[28] O. Bukalo, C. R. Pinard, S. Silverstein et al., "Prefrontal inputs to the amygdala instruct fear extinction memory formation," Sci Adv, vol. 1, no. 6, article e1500251, 2015.

[29] R. R. Rozeske, A. Der-Avakian, S. T. Bland, J. T. Beckley, L. R. Watkins, and S. F. Maier, "The Medial Prefrontal Cortex Regulates the Differential Expression of Morphine-Conditioned Place Preference Following a Single Exposure to Controllable or Uncontrollable Stress," Neuropsychopharmacology, vol. 34, no. 4, pp. 834-843, 2009.

[30] J. P. Mackay, M. Bompolaki, M. R. DeJoseph, S. D. Michaelson, J. H. Urban, and W. F. Colmers, "NPY2Receptors Reduce Tonic Action Potential-Independent GABABCurrents in the Basolateral Amygdala," Journal of Neuroscience, vol. 39, no. 25, pp. 4909-4930, 2019.

[31] A. Szőnyi, K. E. Sos, R. Nyilas et al., "Brainstem nucleus incertus controls contextual memory formation," Science, vol. 364, no. 6442, article eaaw0445, 2019.

[32] R. S. Duman, G. Sanacora, and J. H. Krystal, "Altered Connectivity in Depression: GABA and Glutamate Neurotransmitter Deficits and Reversal by Novel Treatments," Neuron, vol. 102, no. 1, pp. 75-90, 2019.

[33] Y. Kubota, N. Shigematsu, F. Karube et al., "Selective coexpression of multiple chemical markers defines discrete populations of neocortical GABAergic neurons," Cerebral Cortex, vol. 21, no. 8, pp. 1803-1817, 2011.

[34] S. H. Hendry, E. G. Jones, J. DeFelipe, D. Schmechel, C. Brandon, and P. C. Emson, "Neuropeptide-containing neurons of the cerebral cortex are also GABAergic," Proceedings of the National Academy of Sciences of the United States of America, vol. 81, no. 20, pp. 6526-6530, 1984.

[35] R. Tremblay, S. Lee, and B. Rudy, "GABAergic Interneurons in the Neocortex: From Cellular Properties to Circuits," Neuron, vol. 91, no. 2, pp. 260-292, 2016.

[36] B. Czéh, Z. K. Kalangyáné Varga, K. Henningsen, G. L. Kovács, A. Miseta, and O. Wiborg, "Chronic stress reduces the number of GABAergic interneurons in the adult rat 
hippocampus, dorsal-ventral and region-specific differences," Hippocampus, vol. 25, no. 3, pp. 393-405, 2015.

[37] C. Giachino, N. Canalia, F. Capone et al., "Maternal deprivation and early handling affect density of calcium binding protein-containing neurons in selected brain regions and emotional behavior in periadolescent rats," Neuroscience, vol. 145, no. 2, pp. 568-578, 2007.

[38] H. J. Krugers and M. Joels, "Long-lasting Consequences of Early Life Stress on Brain Structure, Emotion and Cognition," vol. 18 of Current Topics in Behavioral Neurosciences, pp. 81-92, 2014.

[39] T. A. Kosten, H. J. Lee, and J. J. Kim, "Early life stress impairs fear conditioning in adult male and female rats," Brain Research, vol. 1087, no. 1, pp. 142-150, 2006.

[40] H. González-Pardo, J. L. Arias, G. Vallejo, and N. M. Conejo, "Influence of environmental enrichment on the volume of brain regions sensitive to early life stress by maternal separation in rats," Psicothema, vol. 31, no. 1, pp. 46-52, 2019.

[41] Z. Varga, D. Csabai, A. Miseta, O. Wiborg, and B. Czéh, "Chronic stress affects the number of GABAergic neurons in the orbitofrontal cortex of rats," Behavioural Brain Research, vol. 316, pp. 104-114, 2017.

[42] H. Pantazopoulos, J. T. Wiseman, M. Markota, L. Ehrenfeld, and S. Berretta, "Decreased Numbers of SomatostatinExpressing Neurons in the Amygdala of Subjects With Bipolar Disorder or Schizophrenia: Relationship to Circadian Rhythms," Biological Psychiatry, vol. 81, no. 6, pp. 536-547, 2017.

[43] L. C. Lin and E. Sibille, "Somatostatin, neuronal vulnerability and behavioral emotionality," Molecular Psychiatry, vol. 20, no. 3, pp. 377-387, 2015.

[44] A. Faron-Górecka, M. Kuśmider, M. Kolasa et al., "Chronic mild stress alters the somatostatin receptors in the rat brain," Psychopharmacology, vol. 233, no. 2, pp. 255-266, 2016.

[45] A. Faron-Górecka, M. Kuśmider, J. Solich et al., "Regulation of somatostatin receptor 2 in the context of antidepressant treatment response in chronic mild stress in rat," Psychopharmacology, vol. 235, no. 7, pp. 2137-2149, 2018.

[46] T. Fuchs, S. J. Jefferson, A. Hooper, P. H. P. Yee, J. Maguire, and B. Luscher, "Disinhibition of somatostatin-positive GABAergic interneurons results in an anxiolytic and antidepressant-like brain state," Molecular Psychiatry, vol. 22, no. 6, pp. 920-930, 2017.

[47] C. Miao, Q. Cao, M. B. Moser, and E. I. Moser, "Parvalbumin and Somatostatin Interneurons Control Different SpaceCoding Networks in the Medial Entorhinal Cortex," Cell, vol. 171, no. 3, pp. 507-521.e17, 2017.

[48] K. L. Wahlstrom, M. L. Huff, E. B. Emmons et al., "Basolateral Amygdala Inputs to the Medial Entorhinal Cortex Selectively Modulate the Consolidation of Spatial and Contextual Learning," The Journal of Neuroscience, vol. 38, no. 11, pp. 26982712, 2018.

[49] D. Kim, H. Jeong, J. Lee et al., "Distinct Roles of Parvalbumin- and Somatostatin-Expressing Interneurons in Working Memory," Neuron, vol. 92, no. 4, pp. 902-915, 2016.

[50] R. Marek, J. Jin, T. D. Goode et al., "Hippocampus-driven feed-forward inhibition of the prefrontal cortex mediates relapse of extinguished fear," Nature Neuroscience, vol. 21, no. 3, pp. 384-392, 2018.

[51] J. A. Brown, T. S. Ramikie, M. J. Schmidt et al., "Inhibition of parvalbumin-expressing interneurons results in complex behavioral changes," Molecular Psychiatry, vol. 20, no. 12, pp. 1499-1507, 2015.

[52] J. Courtin, F. Chaudun, R. R. Rozeske et al., "Prefrontal parvalbumin interneurons shape neuronal activity to drive fear expression," Nature, vol. 505, no. 7481, pp. 92-96, 2014.

[53] E. K. Lucas, A. M. Jegarl, H. Morishita, and R. L. Clem, "Multimodal and Site-Specific Plasticity of Amygdala Parvalbumin Interneurons after Fear Learning," Neuron, vol. 91, no. 3, pp. 629-643, 2016.

[54] I. Férézou, B. Cauli, E. L. Hill, J. Rossier, E. Hamel, and B. Lambolez, "5-HT3Receptors Mediate Serotonergic Fast Synaptic Excitation of Neocortical Vasoactive Intestinal Peptide/Cholecystokinin Interneurons," The Journal of Neuroscience, vol. 22, no. 17, pp. 7389-7397, 2002.

[55] M. Morales and C. Backman, "Coexistence of serotonin 3 (5HT3) and CB1 cannabinoid receptors in interneurons of hippocampus and dentate gyrus," Hippocampus, vol. 12, no. 6, pp. 756-764, 2002.

[56] M. Morales, S. D. Wang, O. Diaz-Ruiz, and D. Hyun-Jin Jho, "Cannabinoid CB1 receptor and serotonin 3 receptor subunit A (5-HT3A) are co-expressed in GABA neurons in the rat telencephalon," The Journal of Comparative Neurology, vol. 468, no. 2, pp. 205-216, 2004.

[57] M. E. Bowers, D. C. Choi, and K. J. Ressler, "Neuropeptide regulation of fear and anxiety: implications of cholecystokinin, endogenous opioids, and neuropeptide Y," Physiology \& Behavior, vol. 107, no. 5, pp. 699-710, 2012.

[58] A. N. Suarez, T. M. Hsu, C. M. Liu et al., "Gut vagal sensory signaling regulates hippocampus function through multiorder pathways," Nature Communications, vol. 9, no. 1, p. $2181,2018$.

[59] L. Medrihan, Y. Sagi, Z. Inde et al., "Initiation of Behavioral Response to Antidepressants by Cholecystokinin Neurons of the Dentate Gyrus," Neuron, vol. 95, no. 3, pp. 564576.e4, 2017.

[60] P. Zwanzger, D. Eser, S. Aicher et al., "Effects of Alprazolam on Cholecystokinin-Tetrapeptide-Induced Panic and Hypothalamic-Pituitary-Adrenal-Axis Activity: A PlaceboControlled Study," Neuropsychopharmacology, vol. 28, no. 5, pp. 979-984, 2003.

[61] S. A. Booker, D. Althof, A. Gross et al., "KCTD12 Auxiliary Proteins Modulate Kinetics of GABABReceptor-Mediated Inhibition in Cholecystokinin-Containing Interneurons," Cerebral Cortex, vol. 27, no. 3, pp. 2318-2334, 2017.

[62] P. D. Whissell, J. Y. Bang, I. Khan et al., "Selective Activation of Cholecystokinin-Expressing GABA (CCK-GABA) Neurons Enhances Memory and Cognition," eNeuro, vol. 6, no. $1,2019$.

[63] L. E. Kuo, J. B. Kitlinska, J. U. Tilan et al., "Neuropeptide Y acts directly in the periphery on fat tissue and mediates stress-induced obesity and metabolic syndrome," Nature Medicine, vol. 13, no. 7, pp. 803-811, 2007.

[64] X. Zhang and A. N. van den Pol, "Hypothalamic arcuate nucleus tyrosine hydroxylase neurons play orexigenic role in energy homeostasis," Nature Neuroscience, vol. 19, no. 10, pp. 1341-1347, 2016.

[65] Y. Nakamura, Y. Yanagawa, S. F. Morrison, and K. Nakamura, "Medullary Reticular Neurons Mediate Neuropeptide Y-Induced Metabolic Inhibition and Mastication," Cell Metabolism, vol. 25, no. 2, pp. 322-334, 2017. 
[66] Q. Wu, Z. Zhao, and P. Shen, "Regulation of aversion to noxious food by Drosophila neuropeptide Y- and insulin-like systems," Nature Neuroscience, vol. 8, no. 10, pp. 1350$1355,2005$.

[67] C. K. Ip, L. Zhang, A. Farzi et al., "Amygdala NPY Circuits Promote the Development of Accelerated Obesity under Chronic Stress Conditions," Cell Metabolism, vol. 30, no. 1, pp. 111-128.e6, 2019.

[68] Z. Zhou, G. Zhu, A. R. Hariri et al., "Genetic variation in human NPY expression affects stress response and emotion," Nature, vol. 452, no. 7190, pp. 997-1001, 2008.

[69] F. Reichmann and P. Holzer, "Neuropeptide Y: A stressful review," Neuropeptides, vol. 55, pp. 99-109, 2016.

[70] M. Banasr, A. Lepack, C. Fee et al., "Characterization of GABAergic marker expression in the chronic unpredictable stress model of depression," Chronic Stress, vol. 1, article 247054701772045, no. 1, 2017.

[71] W. H. Rostene, "Neurobiological and neuroendocrine functions of the vasoactive intestinal peptide (VIP)," Progress in Neurobiology, vol. 22, no. 2, pp. 103-129, 1984.

[72] P. J. Magistretti, J. H. Morrison, W. J. Shoemaker, V. Sapin, and F. E. Bloom, "Vasoactive intestinal polypeptide induces glycogenolysis in mouse cortical slices: a possible regulatory mechanism for the local control of energy metabolism," Proceedings of the National Academy of Sciences of the United States of America, vol. 78, no. 10, pp. 6535-6539, 1981.

[73] M. L. H. J. Hermes, M. Kolaj, P. Doroshenko, E. Coderre, and L. P. Renaud, "Effects of VPAC2 Receptor Activation on Membrane Excitability and GABAergic Transmission in Subparaventricular Zone Neurons Targeted by Suprachiasmatic Nucleus," Journal of Neurophysiology, vol. 102, no. 3, pp. 1834-1842, 2009.

[74] X. Zhou, M. Rickmann, G. Hafner, and J. F. Staiger, "Subcellular Targeting of VIP Boutons in Mouse Barrel Cortex is Layer-Dependent and not Restricted to Interneurons," Cerebral Cortex, vol. 27, no. 11, pp. 5353-5368, 2017.

[75] D. Cunha-Reis, A. M. Sebastião, K. Wirkner, P. Illes, and J. A. Ribeiro, "VIP enhances both pre- and postsynaptic GABAergic transmission to hippocampal interneurones leading to increased excitatory synaptic transmission to CA1 pyramidal cells," British Journal of Pharmacology, vol. 143, no. 6, pp. 733-744, 2004.

[76] C. Dávid, A. Schleicher, W. Zuschratter, and J. F. Staiger, "The innervation of parvalbumin-containing interneurons by VIP-immunopositive interneurons in the primary somatosensory cortex of the adult rat," The European Journal of Neuroscience, vol. 25, no. 8, pp. 2329-2340, 2007.

[77] D. Cunha-Reis, J. A. Ribeiro, R. F. M. de Almeida, and A. M. Sebastião, "VPAC1and VPAC2receptor activation on GABA release from hippocampal nerve terminals involve several different signalling pathways," British Journal of Pharmacology, vol. 174, no. 24, pp. 4725-4737, 2017.

[78] O. T. Korkmaz, N. Tunçel, M. Tunçel, E. M. Öncü, V. Şahintürk, and M. Çelik, "Vasoactive Intestinal Peptide (VIP) Treatment of Parkinsonian Rats Increases Thalamic Gamma-Aminobutyric Acid (GABA) Levels and Alters the Release of Nerve Growth Factor (NGF) by Mast Cells," Journal of Molecular Neuroscience, vol. 41, no. 2, pp. 278-287, 2010.

[79] I. H. Yelkenli, E. Ulupinar, O. T. Korkmaz et al., "Modulation of Corpus Striatal Neurochemistry by Astrocytes and Vaso- active Intestinal Peptide (VIP) in Parkinsonian Rats," Journal of Molecular Neuroscience, vol. 59, no. 2, pp. 280-289, 2016.

[80] B. Bonaz, T. Bazin, and S. Pellissier, "The Vagus Nerve at the Interface of the Microbiota-Gut-Brain Axis," Frontiers in Neuroscience, vol. 12, 2018.

[81] J. W. Maniscalco and L. Rinaman, "Vagal Interoceptive Modulation of Motivated Behavior," Physiology, vol. 33, no. 2, pp. 151-167, 2018.

[82] M. Klarer, U. Weber-Stadlbauer, M. Arnold, W. Langhans, and U. Meyer, "Abdominal vagal deafferentation alters affective behaviors in rats," Journal of Affective Disorders, vol. 252, pp. 404-412, 2019.

[83] M. Klarer, J. P. Krieger, J. Richetto et al., “Abdominal Vagal Afferents Modulate the Brain Transcriptome and Behaviors Relevant to Schizophrenia," The Journal of Neuroscience, vol. 38, no. 7, pp. 1634-1647, 2018.

[84] M. Klarer, M. Arnold, L. Gunther, C. Winter, W. Langhans, and U. Meyer, "Gut Vagal Afferents Differentially Modulate Innate Anxiety and Learned Fear," Journal of Neuroscience, vol. 34, no. 21, pp. 7067-7076, 2014.

[85] E. K. Williams, R. B. Chang, D. E. Strochlic, B. D. Umans, B. B. Lowell, and S. D. Liberles, "Sensory Neurons that Detect Stretch and Nutrients in the Digestive System," Cell, vol. 166, no. 1, pp. 209-221, 2016.

[86] S. Kim, S. H. Kwon, T. I. Kam et al., "Transneuronal Propagation of Pathologic $\alpha$-Synuclein from the Gut to the Brain Models Parkinson's Disease," Neuron, vol. 103, no. 4, pp. 627-641.e7, 2019.

[87] G. D'Agostino, D. J. Lyons, C. Cristiano et al., "Appetite controlled by a cholecystokinin nucleus of the solitary tract to hypothalamus neurocircuit," Elife, vol. 5, 2016.

[88] L. C. Conrad and D. W. Pfaff, "Efferents from medial basal forebrain and hypothalamus in the rat. II. An autoradiographic study of the anterior hypothalamus," The Journal of Comparative Neurology, vol. 169, no. 2, pp. 221-261, 1976.

[89] S. Breit, A. Kupferberg, G. Rogler, and G. Hasler, "Vagus Nerve as Modulator of the Brain-Gut Axis in Psychiatric and Inflammatory Disorders," Frontiers in Psychiatry, vol. 9, 2018.

[90] B. Bonaz, V. Sinniger, and S. Pellissier, "Vagus nerve stimulation: a new promising therapeutic tool in inflammatory bowel disease," Journal of Internal Medicine, vol. 282, no. 1, pp. 4663, 2017.

[91] C. M. Pariante and S. L. Lightman, "The HPA axis in major depression: classical theories and new developments," Trends in Neurosciences, vol. 31, no. 9, pp. 464-468, 2008.

[92] A. Shea, C. Walsh, H. MacMillan, and M. Steiner, "Child maltreatment and HPA axis dysregulation: relationship to major depressive disorder and post traumatic stress disorder in females," Psychoneuroendocrinology, vol. 30, no. 2, pp. $162-$ 178, 2005.

[93] M. H. Greisen, T. G. Bolwig, and G. Wortwein, "Cholecystokinin tetrapeptide effects on HPA axis function and elevated plus maze behaviour in maternally separated and handled rats," Behavioural Brain Research, vol. 161, no. 2, pp. 204212, 2005.

[94] R. Magalhães, A. Novais, D. A. Barrière et al., "A RestingState Functional MR Imaging and Spectroscopy Study of the Dorsal Hippocampus in the Chronic Unpredictable Stress Rat Model," The Journal of Neuroscience, vol. 39, no. 19, pp. 3640-3650, 2019. 
[95] J. M. Staib, R. Della Valle, and D. K. Knox, "Disruption of medial septum and diagonal bands of Broca cholinergic projections to the ventral hippocampus disrupt auditory fear memory," Neurobiology of Learning and Memory, vol. 152, pp. 71-79, 2018.

[96] T. Korotkova, A. Ponomarenko, C. K. Monaghan et al., "Reconciling the different faces of hippocampal theta: The role of theta oscillations in cognitive, emotional and innate behaviors," Neuroscience and Biobehavioral Reviews, vol. 85, pp. 65-80, 2018.

[97] S. S. Chee, J. L. Menard, and H. C. Dringenberg, "Behavioral anxiolysis without reduction of hippocampal theta frequency after histamine application in the lateral septum of rats," Hippocampus, vol. 24, no. 6, pp. 615-627, 2014.

[98] Y. T. Hsiao, P. L. Yi, C. H. Cheng, and F. C. Chang, "Disruption of footshock-induced theta rhythms by stimulating median raphe nucleus reduces anxiety in rats," Behavioural Brain Research, vol. 247, pp. 193-200, 2013.

[99] S. S. Chee, J. L. Menard, and H. C. Dringenberg, "The lateral septum as a regulator of hippocampal theta oscillations and defensive behavior in rats," Journal of Neurophysiology, vol. 113, no. 6, pp. 1831-1841, 2015.

[100] A. Mitra, C. Lenglos, and E. Timofeeva, "Inhibition in the lateral septum increases sucrose intake and decreases anorectic effects of stress," The European Journal of Neuroscience, vol. 41, no. 4, pp. 420-433, 2015.

[101] M. I. Cordero, N. Just, G. L. Poirier, and C. Sandi, "Effects of paternal and peripubertal stress on aggression, anxiety, and metabolic alterations in the lateral septum," European Neuropsychopharmacology, vol. 26, no. 2, pp. 357-367, 2016.

[102] A. Besnard, Y. Gao, M. TaeWoo Kim et al., "Dorsolateral septum somatostatin interneurons gate mobility to calibrate context-specific behavioral fear responses," Nature Neuroscience, vol. 22, no. 3, pp. 436-446, 2019.

[103] G.-W. Zhang, L. Shen, W. Zhong, Y. Xiong, L. I. Zhang, and H. W. Tao, "Transforming Sensory Cues into Aversive Emotion via Septal-Habenular Pathway," Neuron, vol. 99, no. 5, pp. 1016-1028.e5, 2018.

[104] B. G. Dias, J. V. Goodman, R. Ahluwalia, A. E. Easton, R. Andero, and K. J. Ressler, "Amygdala-dependent fear memory consolidation via miR-34a and Notch signaling," Neuron, vol. 83, no. 4, pp. 906-918, 2014.

[105] H. S. Knobloch, A. Charlet, L. C. Hoffmann et al., "Evoked axonal oxytocin release in the central amygdala attenuates fear response," Neuron, vol. 73, no. 3, pp. 553-566, 2012.

[106] J. Waddell, D. A. Bangasser, and T. J. Shors, “The basolateral nucleus of the amygdala is necessary to induce the opposing effects of stressful experience on learning in males and females," Journal of Neuroscience, vol. 28, no. 20, pp. 52905294, 2008.

[107] W. B. Kim and J. H. Cho, "Encoding of Discriminative Fear Memory by Input-Specific LTP in the Amygdala," Neuron, vol. 95, no. 5, pp. 1129-1146.e5, 2017.

[108] A. M. Douglass, H. Kucukdereli, M. Ponserre et al., "Central amygdala circuits modulate food consumption through a positive-valence mechanism," Nature Neuroscience, vol. 20, no. 10, pp. 1384-1394, 2017.

[109] J. Kim, X. Zhang, S. Muralidhar, S. A. LeBlanc, and S. Tonegawa, "Basolateral to Central Amygdala Neural Circuits for Appetitive Behaviors," Neuron, vol. 93, no. 6, pp. 1464-1479.e5, 2017.
[110] F. Mascagni and A. J. McDonald, "A novel subpopulation of 5-HT type $3 \mathrm{~A}$ receptor subunit immunoreactive interneurons in the rat basolateral amygdala," Neuroscience, vol. 144, no. 3, pp. 1015-1024, 2007.

[111] T. Rhomberg, L. Rovira-Esteban, A. Vikór et al., "Vasoactive Intestinal Polypeptide-Immunoreactive Interneurons within Circuits of the Mouse Basolateral Amygdala," The Journal of Neuroscience, vol. 38, no. 31, pp. 6983-7003, 2018.

[112] H. Y. Chu, W. Ito, J. Li, and A. Morozov, "Target-specific suppression of GABA release from parvalbumin interneurons in the basolateral amygdala by dopamine," Journal of Neuroscience, vol. 32, no. 42, pp. 14815-14820, 2012.

[113] E. Likhtik, J. M. Stujenske, M. A. Topiwala, A. Z. Harris, and J. A. Gordon, "Prefrontal entrainment of amygdala activity signals safety in learned fear and innate anxiety," Nature Neuroscience, vol. 17, no. 1, pp. 106-113, 2014.

[114] B. Czéh, I. Vardya, Z. Varga et al., "Long-Term Stress Disrupts the Structural and Functional Integrity of GABAergic Neuronal Networks in the Medial Prefrontal Cortex of Rats," Frontiers in Cellular Neuroscience, vol. 12, 2018.

[115] J. M. McKlveen, R. L. Morano, M. Fitzgerald et al., "Chronic Stress Increases Prefrontal Inhibition: A Mechanism for Stress-Induced Prefrontal Dysfunction," Biological Psychiatry, vol. 80, no. 10, pp. 754-764, 2016.

[116] S. Zhang, M. Xu, T. Kamigaki et al., "Selective attention. Long-range and local circuits for top-down modulation of visual cortex processing," Science, vol. 345, no. 6197, pp. 660-665, 2014.

[117] K. Kakizawa, M. Watanabe, H. Mutoh et al., "A novel GABAmediated corticotropin-releasing hormone secretory mechanism in the median eminence," Science Advances, vol. 2, no. 8, article e1501723, 2016.

[118] I. H. Miklos and K. J. Kovacs, "GABAergic innervation of corticotropin-releasing hormone $(\mathrm{CRH})$-secreting parvocellular neurons and its plasticity as demonstrated by quantitative immunoelectron microscopy," Neuroscience, vol. 113, no. 3, pp. 581-592, 2002.

[119] N. Dedic, C. Kühne, M. Jakovcevski et al., "Chronic CRH depletion from GABAergic, long-range projection neurons in the extended amygdala reduces dopamine release and increases anxiety," Nature Neuroscience, vol. 21, no. 6, pp. 803-807, 2018.

[120] K. M. Engster, L. Frommelt, T. Hofmann et al., "Peripheral injected cholecystokinin- $8 \mathrm{~S}$ modulates the concentration of serotonin in nerve fibers of the rat brainstem," Peptides, vol. 59, pp. 25-33, 2014.

[121] C. W. Roman, S. R. Sloat, and R. D. Palmiter, "A tale of two circuits: CCK(NTS) neuron stimulation controls appetite and induces opposing motivational states by projections to distinct brain regions," Neuroscience, vol. 358, pp. 316-324, 2017.

[122] V. O’Keane, T. G. Dinan, L. Scott, and C. Corcoran, “Changes in Hypothalamic-Pituitary-Adrenal Axis Measures After Vagus Nerve Stimulation Therapy in Chronic Depression," Biological Psychiatry, vol. 58, no. 12, pp. 963-968, 2005.

[123] L. K. Stenman, E. Patterson, J. Meunier, F. J. Roman, and M. J. Lehtinen, "Strain specific stress-modulating effects of candidate probiotics: A systematic screening in a mouse model of chronic restraint stress," Behavioural Brain Research, vol. 379, article 112376, 2020. 
[124] R. Janik, L. A. M. Thomason, A. M. Stanisz, P. Forsythe, J. Bienenstock, and G. J. Stanisz, "Magnetic resonance spectroscopy reveals oral Lactobacillus promotion of increases in brain GABA, N-acetyl aspartate and glutamate," NeuroImage, vol. 125, pp. 988-995, 2016.

[125] R. A. Yunes, E. U. Poluektova, M. S. Dyachkova et al., "GABA production and structure of gadB/gadC genes in Lactobacillus and Bifidobacterium strains from human microbiota," Anaerobe, vol. 42, pp. 197-204, 2016.

[126] P. Strandwitz, K. H. Kim, D. Terekhova et al., "GABA-modulating bacteria of the human gut microbiota," Nature Microbiology, vol. 4, no. 3, pp. 396-403, 2019.

[127] M. Takada, K. Nishida, A. Kataoka-Kato et al., "Probiotic Lactobacillus casei strain Shirota relieves stress-associated symptoms by modulating the gut-brain interaction in human and animal models," Neurogastroenterology and Motility, vol. 28, no. 7, pp. 1027-1036, 2016.

[128] J. R. Kelly, A. P. Allen, A. Temko et al., "Lost in translation? The potential psychobiotic Lactobacillus rhamnosus (JB-1) fails to modulate stress or cognitive performance in healthy male subjects," Brain, Behavior, and Immunity, vol. 61, pp. 50-59, 2017.

[129] Y. M. Ulrich-Lai, S. Fulton, M. Wilson, G. Petrovich, and L. Rinaman, "Stress exposure, food intake and emotional state," Stress, vol. 18, no. 4, pp. 381-399, 2015.

[130] T. Onaka and Y. Takayanagi, "Role of oxytocin in the control of stress and food intake," Journal of Neuroendocrinology, vol. 31, no. 3, article e12700, 2019.

[131] R. Chandra, Y. Wang, R. A. Shahid, S. R. Vigna, N. J. Freedman, and R. A. Liddle, "Immunoglobulin-like domain containing receptor 1 mediates fat-stimulated cholecystokinin secretion," The Journal of Clinical Investigation, vol. 123, no. 8, pp. 3343-3352, 2013.

[132] G. Vaiva, P. Thomas, F. Ducrocq et al., "Low posttrauma GABA plasma levels as a predictive factor in the development of acute posttraumatic stress disorder," Biological Psychiatry, vol. 55, no. 3, pp. 250-254, 2004.

[133] R. Stam, L. M. A. Akkermans, and V. M. Wiegant, “Trauma and the gut: interactions between stressful experience and intestinal function,” Gut, vol. 40, no. 6, pp. 704-709, 1997.

[134] A. Gautam, R. Kumar, N. Chakraborty et al., "Altered fecal microbiota composition in all male aggressor-exposed rodent model simulating features of post-traumatic stress disorder," Journal of Neuroscience Research, vol. 96, no. 7, pp. 13111323, 2018.

[135] J. Pearson-Leary, C. Zhao, K. Bittinger et al., "The gut microbiome regulates the increases in depressive-type behaviors and in inflammatory processes in the ventral hippocampus of stress vulnerable rats," Molecular Psychiatry, vol. 25, no. 5, pp. 1068-1079, 2020.

[136] L. J. Noble, I. J. Gonzalez, V. B. Meruva et al., "Effects of vagus nerve stimulation on extinction of conditioned fear and posttraumatic stress disorder symptoms in rats," Translational Psychiatry, vol. 7, no. 8, article e1217, 2017. 